\title{
Equalization of OFDM by Iterative ICI reduction Methods
}

\author{
Chanderkanta $^{1}$, Dr. Sonika Singh ${ }^{2}$ \\ Post Graduate scholar, ECE, DIT University, Dehradun, India ${ }^{1}$ \\ Associate Professor, ECE, DIT University, Dehradun, India ${ }^{2}$
}

\begin{abstract}
Orthogonal frequency division multiplexing (OFDM) is a method of encoding digital data on multiple carrier frequencies and developed as a popular scheme for $4 \mathrm{G}$ mobile communications. Inter-carrier interference (ICI) is a big challenge for OFDM systems while trying to utilize the full spectrum with zero cyclic prefix. It has been investigated that the ICI in OFDM is almost like the co-channel interference in CDMA and the techniques used there to reduce the interference can be used in OFDM also. The techniques are frequency domain equalizers like Operator perturbation Techniques (OPT) and Serial interference cancellation (SIC) and Parallel interference cancellation technique (PIC) along with Gauss Seidel iterative reduction technique. The aim of this paper is to study the OFDM systems suffering from ICI, due to large Doppler shift associated with high velocity of receiver. The main impact of ICI in an OFDM system is to destroy the orthogonality between subcarrier leads to the introduction of off-diagonal element in the channel matrix, making the traditionally considered diagonal channel matrix inaccurate and increasing the complexity of channel estimation and equalization .In this paper iterative cancellation methods have been studied and compared as they require the inversion of only a small portion of original full matrix and thus the complexity is reduced
\end{abstract}

Keywords: OFDM, ICI, Gauss-Seidel, OPT, PIC and SIC

\section{INTRODUCTION}

As the demand for high speed communication (for $4 \mathrm{G}$ systems up to $1 \mathrm{Gbps}$ ) increases, it needs to ensure the performance should not be degraded and channel bandwidth should be utilized in effective way. In single carrier communication, degradation can be reduced by signal processing at receiving end with techniques like adaptive channel equalization and channel coding. But it is very difficult to use on high data rates as it increases the inherent delay, so alternative approach is Multi carrier modulation technique and OFDM [1] is preferred multi carrier modulation technique. The basic principal of OFDM is to split the channel in to $\mathrm{N}$ number of sub channels and transmission of signal using orthogonal carriers, through these sub channels. OFDM converts frequency selective channel in to flat frequency channel so it can completely eliminate the Inter symbol interference (ISI)[5].This is the major advantage of OFDM.

In parallel data transmission, an available frequency band is divided into several channels by independently modulating a number of carriers of different frequency. Since each channel occupies a relatively narrow frequency band, parallel transmission is effective in combating the effects of amplitude and delay distortion and impulsive noise. During 1960's Chang [15], has given different conditions for spectral limited channels .each carrying a data rate ' $b$ ', may be space ' $b / 2$ ' apart in frequency, which results into no inter channel interference(ICI)[5], which leads to design of OFDM system. Under these conditions, using available bandwidth a total signaling rate close to the Nyquist rate may be achieved through the use of a large number of channels. In OFDM, the multiple subcarriers are orthogonal to each other. If this orthogonality disturbs due to some reason like Doppler shift, or the difference in local oscillator frequency in transmitter and receiver, it causes the inter-channel interference (ICI). There are various techniques to combat the effect of Inter-carrier interference.

\section{Operator Perturbation Technique (OPT)}

The conventional method to combat the effect of ICI and ISI is to adding the cyclic prefix (CP). The drawback of the $\mathrm{CP}$ (length $\mathrm{G}$ symbols) is that it reduces the spectral efficiency to $\frac{N}{N+G}[12]$. This reduction can be eliminated only by omitting the $\mathrm{CP}$. To improve the channel spectral efficiency with zero $\mathrm{CP}$, the inversion of channel impulse matrix for ICI cancellation is done using Operator perturbation technique (OPT) [12], which reduces the Computational complexity also by the $\mathrm{O}\left(\mathrm{n}^{2}\right)$ operations per iteration.

\section{A. OPT algorithm}

OPT is an effective way to invert linear or non-linear operators, and is also called the Jacobi iteration [12]. It was originally introduced by J.C.Canon [12]. This is an iterative method for inversion of OFDM channel matrix $\mathrm{H}$. In standard OPT, a banded matrix $\widehat{H}$ consists of the diagonal and $\mathrm{Q}$ off-diagonals of matrix $\mathrm{H}$ as in is extracted and it is given as

For notational convenience we can write

$$
\begin{aligned}
& Y=H X \\
& X=H^{-1} Y
\end{aligned}
$$

And approximate (1) by

$$
\mathrm{Y}=\widehat{H} \mathrm{X}-\mathrm{C}
$$


$\widehat{\mathrm{H}}$ is the approximate operator whose inverse is easy to and therefore, $\mathrm{X}_{(\infty)}=\mathrm{X}$. Step (7) of the iteration procedure compute, $€$ is the deviation from the exact solution.E.g. $\widehat{H}$ requires a vector-matrix product that needs $\mathrm{O}\left(\mathrm{n}^{2}\right)$ could consist of the diagonal of $\mathrm{H}$ and zero off-diagonal operations. The sufficient condition for OPT to converge elements. The solution of (2) is then found by the is that the matrix $\widehat{H}$ is strictly or irreducibly diagonally following iteration:

$$
\begin{aligned}
& \mathrm{H}= \\
& \left(\begin{array}{lllll}
\mathrm{h}_{1,1} & \mathrm{~h}_{1,2} & \ldots \ldots & \ldots \ldots \ldots \ldots \ldots & \mathrm{h}_{1, \mathrm{n}} \\
\mathrm{h}_{2,1} & \mathrm{~h}_{2,2} & \ldots \ldots & \ldots \ldots \ldots \ldots \ldots & \mathrm{h}_{2, \mathrm{n}} \\
\cdot & \cdot & \ldots \ldots & \ldots \ldots \ldots \ldots \ldots & \cdot \\
\cdot & \cdot & \ldots \ldots & \ldots \ldots \ldots \ldots \ldots & \cdot \\
\cdot & \cdot & & & \cdot \\
\cdot \mathrm{h}_{\mathrm{n}, 1} & \cdot & & & \\
\mathrm{h}_{\mathrm{n}, 2} & \ldots \ldots \ldots & \ldots \ldots \ldots \ldots \ldots & \mathrm{h}_{\mathrm{n}, \mathrm{n}}
\end{array}\right) \\
& \widehat{H}=\left(\begin{array}{llll}
\mathrm{h}_{1,1} & 0 & 0 & 0 \ldots \ldots \ldots 0 \\
\mathrm{~h}_{2,1} & \mathrm{~h}_{2,2} & 0 & 0 \ldots \ldots \ldots 0 \\
\mathrm{~h}_{3,1} & \mathrm{~h}_{3,2} & \mathrm{~h}_{3,3} & 0 \ldots \ldots \ldots 0 \\
\cdot & \cdot & \cdot & 0 \ldots \ldots \ldots 0 \\
\cdot & \cdot & \cdot & 0 \ldots \ldots \ldots 0 \\
\dot{h_{n}-1,1} & \mathrm{~h}_{\mathrm{n}-1,2} & \dot{\mathrm{h}}_{\mathrm{n}-1,3} & 0 \ldots \ldots .0 \\
\mathrm{~h}_{\mathrm{n}, 1} & \mathrm{~h}_{\mathrm{n}, 2} & \mathrm{~h}_{\mathrm{n}, 3} & \ldots \ldots . \mathrm{h}_{\mathrm{n}, \mathrm{n}}
\end{array}\right) \\
& \mathrm{X}_{(0)}=\widehat{\mathrm{H}}^{-1} \cdot \mathrm{Y} \\
& \epsilon_{(0)}=(\widehat{H}-H) X_{(0)} \\
& X_{(i+1)}=\widehat{H}^{-1} \cdot\left(\epsilon_{(i)}+Y\right) \\
& \epsilon_{(\mathrm{i}+1)}=(\widehat{\mathrm{H}}-\mathrm{H}) \mathrm{X}_{(\mathrm{i}+1)}
\end{aligned}
$$

Where subscript $\mathrm{i}$ denote $\mathrm{i}^{\text {th }}$ iteration, and equation (4) and (5) are initialization steps. Step (6) and (7) are repeated until reached a criterion. Substituting step (6) in step (7) gives:

$$
\mathrm{X}_{(\mathrm{i}+1)}=\mathrm{X}_{(\mathrm{i})}+\widehat{\mathrm{H}}^{-1}(\mathrm{Y}-\mathrm{HX}(\mathrm{i})
$$

where $\widehat{\mathrm{H}}^{-1}\left(\mathrm{Y}-\mathrm{HX}_{(\mathrm{i})}\right)$ can be represent as $\mathrm{e}_{(\mathrm{x})}$ and $\mathrm{e}_{(\mathrm{x})}$ is the error of old solution $\mathrm{X}_{(\mathrm{i})}$. If $\mathrm{X}_{(\mathrm{i})}$ converges to $\mathrm{X}_{(\infty)}$, then

$$
\begin{aligned}
& X_{(\infty)}=\widehat{H}^{-1} \cdot\left(\mathrm{C}_{(\infty)}+\mathrm{Y}\right) \\
& \epsilon_{(\infty)}=(\widehat{\mathrm{H}}-\mathrm{H}) \mathrm{X}_{(\infty)} \\
& \mathrm{X}_{(\infty)}=\widehat{\mathrm{H}}^{-1} \cdot\left((\widehat{\mathrm{H}}-\mathrm{H}) \cdot \mathrm{X}_{(\infty)}+\mathrm{Y}\right) \\
& =\mathrm{X}_{(\infty)}+\widehat{H}^{-1} \cdot\left(\mathrm{Y}-\mathrm{H} \cdot \mathrm{X}_{(\infty)}\right) \\
& \Rightarrow 0=\widehat{\mathrm{H}}^{-1} \cdot\left(\mathrm{Y}-\mathrm{H} \cdot \mathrm{X}_{(\infty)}\right)
\end{aligned}
$$

This means that if det $\left\{\widehat{\mathbf{H}}^{-1}\right\} \neq 0$, then we get the solution

$$
\mathrm{Y}-\mathrm{HX}_{(\infty)}=0
$$
dominant, that is within each row the absolute value of the diagonal term is greater than the sum of absolute values of other terms, given as:

$$
|h i, i|>\sum_{j \neq i}|h i, j|
$$

With the help of OPT technique, Channel matrix $\mathrm{H}$ inversion complexity reduces a lot. It requires $\mathrm{O}\left(\mathrm{n}^{2}\right)$ operations while a conventional equation solver based on matrix inversion requires $\mathrm{O}\left(\mathrm{n}^{3}\right)$ operations.

\section{GAUSS-SEIDEL ITERATION}

A similar method to Gauss-Seidel Iteration OPT is called the Gauss-Seidel iteration, which is named after German mathematicians Carl Friedrich Gauss and Philipp Ludwig von Seidel [11]. It is an improved version of OPT in respect of its memory efficiency, as it does not require additional memory to store $X_{(i)}$.As with the OPT method, although Gauss-Seidel iteration can be applied to matrices with non-zero diagonals, convergence is guaranteed only when the main diagonal is strictly or irreducibly diagonally dominant. The main difference to Jacobi iteration is that when forming the sparse matrix, Gauss-Seidel iteration uses the lower triangle of the matrix $\mathrm{H}$ instead of using a banded matrix as in OPT.

\section{B. Gauss-Seidel algorithm}

In this iteration method, the channel matrix $\mathrm{H}$ decomposed into three sub-matrixes noted as

$$
\mathrm{H}=\mathrm{D}+\mathrm{E}+\mathrm{F}=\mathrm{D}(\mathrm{I}+\mathrm{L}+\mathrm{U})
$$

Where $\mathrm{E}, \mathrm{L}$ is strict lower triangular matrixes and $\mathrm{F}, \mathrm{U}$ is strict upper triangular matrixes and $\mathrm{D}$ is a diagonal matrix. For the data vector $X=\left[X_{0}, X_{1} \ldots X_{N-1}\right]$, Jacobi type iteration is used to evaluate $X_{(k+1)}$, for already evaluated $X_{i}^{(k+1) \ldots} X_{s}{ }^{(k+1)}$ evaluation could be done on the $s^{\text {th }}$ row of $\mathrm{LX}^{(\mathrm{K}+1)}$ in matrix notation

$$
\begin{aligned}
& X^{(k+1)}=L^{(k+1)}+U^{(k)}+D^{-1} Y \\
& X^{(k+1)}=(I-L)^{-1}\left(U X^{(k)}+D^{-1} Y\right)
\end{aligned}
$$

The iteration matrix (I- L $)^{-1} \mathrm{U}$ is called Gauss Seidel iteration matrix [13]. The algorithm can be summarized in these steps:

$>\quad$ Initial guess of the unknown Data vector $\mathrm{X}^{(0)}=0$. $>\quad$ Using the equation to compare $\mathrm{X}^{(\mathrm{k}+1)}$. Once $\mathrm{X}_{\mathrm{i}}{ }^{(\mathrm{k}+1)}, \mathrm{i}=0,1 \ldots \ldots \ldots, \mathrm{N}-1$ in each iterative step as soon as the part of the new data vector have been calculated.

After each iteration, examine if the errors are less than tolerance

$$
\epsilon_{i}=\left|X_{i}^{(k+1)}-X_{i}^{(k)}\right|, i=0,1, \ldots \ldots, N-1
$$

If the error is not smaller than the tolerance then continue the iteration. 
The main idea of this algorithm is to update the data signal-to interference ratio (SIR), is large. That is the case vector $X^{(k)}$ in each iterative vector step as soon as the part for good channels with small delay spread [14]. of the new data vector $X^{(k+1)}$ have been calculated, Performance can be further improved if $c$ is increased therefore ,the convergence of the algorithm can be from one iteration step to the next. In the beginning, accelerated effectively.

\section{PARALLEL INTERFERENCE CANCELLATION TECHNIQUE(PIC)}

The Jacobi Iteration and Gauss-Seidel iteration uses complete linear operations to achieve interference cancellation. Non-linear operation can also be incorporated with linear operations to achieve improved accuracy. Parallel Interference cancellation (PIC) and Serial Interference Cancellation (SIC) are two of this kind. PIC uses a similar approach to OPT, where the cancellation process takes place in a symbol wise way for OFDM systems.

\section{PIC algorithm}

For initialization, calculate a first estimate,

$\mathrm{X}_{\mathrm{i}}^{(0)}=(\operatorname{diag~H})^{-1} \cdot \mathrm{Y}$

Here, diagH is the diagonal of $\mathrm{H}$ only and can be inverted with computational effort $\mathrm{O}(\mathrm{n})$. The in step $\mathrm{n}$ is

$$
I^{(n)}=\mathrm{HX}^{(\mathrm{n}-1)}-\operatorname{diagH} \mathrm{X}^{(\mathrm{n}-1)}
$$
interference $\mathrm{I}^{(\mathrm{n})}$

where we omitted the block index sub i for notational convenience. The updated interference-cancelled received symbol vector $\mathrm{Y}^{(\mathrm{n})}$ is simply

$\mathrm{Y}^{(\mathrm{n})}=\mathrm{Y}-\mathrm{I}^{(\mathrm{n})}$

Hence the updated estimate of the transmitted symbol on subcarrier $\mathrm{k}$ is

$$
\mathrm{X}_{\mathrm{k}}^{(\mathrm{n})}=\mathrm{Y}_{\mathrm{k}}^{(\mathrm{n}) / \mathrm{H}_{\mathrm{k}}}
$$

where we equalize the interference-cancelled received symbol by the channel response on the frequency of subcarrier k. This algorithm is called "parallel"[14], because Eqs. (18) and (19) can be applied to all channels k $=0 \ldots \mathrm{n}-1$ simultaneously by a vector-matrix calculus. Due to the vector matrix multiplication, the computational complexity of the algorithm is $\mathrm{O}\left(\mathrm{n}^{2}\right)$. Before entering the next round of iterations; the estimated symbols can be processed in a nonlinear fashion. In the simplest case, they could be forced to the nearest symbol of the used alphabet. More generally

$$
\widehat{\mathrm{X}}_{\mathrm{k}}^{(\mathrm{n})}=f \mathrm{X}_{\mathrm{k}}^{(\mathrm{n})}
$$

Where $f($.$) is the nonlinear decision function, like for$ BPSK [2] hyperbolic tangent function,

$$
\widehat{\mathrm{X}}_{\mathrm{k}}^{(\mathrm{n})}=\tanh \left(\mathrm{c} \cdot \mathrm{X}_{\mathrm{k}}^{(\mathrm{n})}\right) \text {. }
$$

because far-off estimates are forced to \pm 1 and thus reduced in their impact on the next cancellation stage, while estimates with small amplitudes are more or less unchanged. The factor $\mathrm{c}$ in the hyperbolic tangent function controls the slope near zero. Small c (e.g., c $<1$ ) are appropriate if the estimates are not yet reliable. Large c (c > 10) are appropriate for accurate estimates, e.g., if the decisions are still unreliable so that small $\mathrm{c}$ is appropriate. At the end of the iteration, the decision function approximates the signum function, and all decisions are in the set $\{ \pm 1\}$. This technique stems from an optimization technique called "simulated annealing" [14] and avoids the convergence of the MSE to a local minimum. The error vector and the MSE in the receiver are defined as

$$
\begin{aligned}
& \mathrm{E}=\mathrm{Y}-\mathrm{H} . \hat{X}_{\mathrm{i}} \\
& \mathrm{MSE}=\|\in\|
\end{aligned}
$$

The iteration stops after the MSE falls below a predefined threshold or the number of iterations reaches a predefined maximum number.

\section{SERIAL INTERFERENCE CANCELLATION (SIC)}

Serial Interference Cancellation (SIC) is an alternative to PIC and cancels the interference in a subcarrier-wise form. The reason for having SIC is to avoid long processing delays as some subcarriers have smaller Signal to interference ratio (SIR) which requires more iteration while others have larger SIR and convergence may be achieved after only a few iterations. The idea of SIC is to order subcarriers by their respective SIRs, and then process those strong sub-channels with higher SIRs first. By doing so several symbols of one subcarrier can be processed simultaneously and therefore processing delays can be reduced. Using SIC will ensure that high reliability channels (i.e. with high SIR) are processed before the weak channels. In each iteration the subcarriers are processed in the order of their corresponding SIR that was calculated beforehand. The algorithm for SIC is similar to PIC. The main advantage of SIC is that the reduction of long processing delays and that the processing of subcarriers can be distinguished by their channel qualities.

\section{SIC algorithm}

First define a Signal to Interference Ratio (SIR) for the sub channel $\mathrm{k}$ as

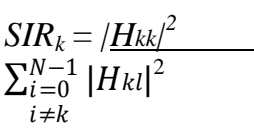

We then order the sub-channels by their SIR. This ensures that high reliable channels (i.e., with high SIR) are processed before the weak channels. In each iteration step (outer loop), the sub-channels are processed in the order of their corresponding SIR (inner loop) that was calculated beforehand for the actual channel situation. For each subchannel $\mathrm{k}$ of iteration step $\mathrm{i}$, the interference

$$
\mathrm{I}^{(\mathrm{n})}=\sum_{\substack{i=0 \\ i \neq k}}^{N c-1}\left|H_{k l}\right| \hat{X}_{\mathrm{i}}{ }^{(\mathrm{n})}
$$

is computed and subtracted. The computational effort is again $\mathrm{O}\left(\mathrm{n}^{2}\right)$. The rest of the algorithm is similar to 
PIC.The major advantage of SIC compared to PIC is that within a single iteration step, the impact of estimated Symbols on low-SIR carriers are considered immediately.

\section{COMPARISON BETWEEN OPT , GAUSS} SEIDEL, PIC AND SIC METHODS

\begin{tabular}{|c|c|c|c|c|}
\hline & $O P T$ & GAUSS & PIC & SIC \\
\hline Types & Linear & Linear & $\begin{array}{l}\text { Non- } \\
\text { linear }\end{array}$ & $\begin{array}{l}\text { Non- } \\
\text { linear }\end{array}$ \\
\hline $\begin{array}{l}\text { Complex } \\
\text { ity } \\
\text { reduction }\end{array}$ & $O\left(N^{2}\right)$ & $O\left(N^{2}\right)$ & $O\left(N^{2}\right)$ & $O\left(N^{2}\right)$ \\
\hline $\begin{array}{l}\text { Performa } \\
\text { nce }\end{array}$ & $\begin{array}{l}\text { Better } \\
\text { than } \\
\text { conven } \\
\text { ti-onal } \\
\text { matrix } \\
\text { inversi } \\
\text { on } \\
\text { techniq } \\
\text { ue }\end{array}$ & $\begin{array}{l}\text { Better than } \\
\text { OPT in } \\
\text { respect of its } \\
\text { memory } \\
\text { efficiency, as } \\
\text { it does not } \\
\text { require } \\
\text { additional } \\
\text { memory to } \\
\text { store } X(i)\end{array}$ & $\begin{array}{l}\text { Symbol } \\
\text { processi } \\
\text { ng is } \\
\text { parallel } \\
\text { so } \\
\text { process } \\
\text { delay is } \\
\text { less. }\end{array}$ & $\begin{array}{l}\text { Symbol } \\
\text { processing } \\
\text { is serially, } \\
\text { but SIC is } \\
\text { still better } \\
\text { than PIC by } \\
\text { a factor of } \\
2-8 \\
\text { in the } \\
\text { BER.[14] }\end{array}$ \\
\hline
\end{tabular}

\section{CONCLUSIONS}

Inter-carrier interference originates from dispersive channels, pulse shaping, or a missing cyclic prefix and has to be equalized for reliably data transmission. The main impact of ICI is loss of orthogonality between subchannels and it leads the off diagonal elements in channel matrix and make it inaccurate and increasing the complexity of channel estimation and equalization. Matrix inversion is traditional method to remove the ICI. Compared to direct matrix inversion techniques that requires $\mathrm{O}\left(\mathrm{n}^{3}\right)$ operations, iterative interference cancellation only needs $\mathrm{O}\left(\mathrm{n}^{2}\right)$ and time variant channels also can be equalized in iterative methods. In this paper, iterative methods have been studied, namely OPT, GaussSeidel iteration, PIC and SIC, all have similar iterative algorithms but their method of constructing the sparse matrix to be inverted is different. It is however noticed that as they treat all subcarriers equally, their method of constructing the matrix is not optimized and will thus limit the accuracy of the cancellation performance.

\section{REFERENCES}

[1] L. Cimini, "Analysis and Simulation of a Digital Mobile Channel Using Orthogonal Frequency Division Multiplexing," IEEE Transactions on Communications, vol. 33, no. 7, pp. 665-675, July 1985

[2] D. Raphaeli, "Iterative Co-channel Interference Cancellation in Synchronous CDMA on a Frequency Selective Channel," in 5th IEEE International Conference on Universal Personal Communications, Cambridge, MA, pp. 336-40,1996.

[3] A.F. Molisch, M. Toeltsch, and S. Vermani, "Iterative Methods for Cancellation of Intercarrier Interference in OFDM systems," ieee transactions on vehicular technology, vol. 56, no. 4, pp. 2158-2167, july 2007

[4] Andreas F. Molisch "Orthogonal frequency division multiplexing" Chapter 19, Wireless Communications, Second Edition (C) 2011 John Wiley \& Sons Ltd. ISBN: 978-0-470-74187-0

[5] Shaoping Chen and Cuitao Zhu "ICI and ISI Analysis and Mitigation for OFDM Systems with Insufficient Cyclic Prefix in Time-Varying Channels" IEEE Transactions on Consumer Electronics, Vol. 50, no. 1, pp. 78-83,, Feburary 2004 